POS PROCEEDINGS

\title{
Concluding remarks - 1
}

\section{G.S.Bisnovatyi-Kogan*}

Space Research Institute, Profsoyuznaya str. 84/32, Moscow 117997, Russia, and National Research Nuclear University "MEPHI", Kashirskoye Shosse, 31, Moscow 115409, Russia E-mail: gkogandiki.rssi.ru

Frontier Research in Astrophysics - II

23-28 May 2016

Mondello (Palermo), Italy

${ }^{*}$ Speaker. 
The TOP news discussed on the Workshop are

1. Discovery of gravitational wave signal by LIGO

2. Discovery of Higgs boson on LHC

Both discoveries were expected for a long time,

Both were done on highest technology instruments, unique and very expensive

Both have a fundamental character, confirming the basic suggestions of the modern physics

But the differences between them are also important

*) Gravitational waves have been discovered by observations of binary pulsars around 1990 in full accordance with General Relativity, so GW discovery in the Earth laboratory is an advantage mainly by the technology, than by the fundamental physics.

Discovery of the Higgs boson, if confirmed, is really fundamental, because it is the base of the particle physics, "the particle of God", on which existence is build all complicated constructions of modern particle theories.

*) The second difference is connected with the conditions of these discoveries. The Higgs boson was produced in LHC, and its search will be prolonged until the final result will become fully reliable.

The gravitational wave burst is a unique transient event which never will be repeated from the same object. The properties of a possible progenitor are unusual, which never has been observed before. Higher frequency gravitational wave signal is really expected from NS merging. This particular signal could remain highly probable, but not definite, like the first BH object in Cyg X-1 remains as a "BH candidate" because of low value of the mass function.

Another outstanding experiment now in operation is Ice Cube on the South Pole. It registered weakly interacting particles at low background in a huge volume of $1 \mathrm{~km}^{3}$, which may be substantially increased in near future. This experiment is similar to Superkamiokande in Japan, where instead of water there is ice. The interpretation of the results in not yet finished, but we may expect important progress in high energy physics and CR origin.

We heard a very impressive list of achievements of HST during its long carrier. Almost all main discoveries of last about 20 years have been done with help of HST: from dark energy in cosmology until exoplanets. It seems to be the most successful scientific space mission. Hope that similar mission planned in Russia would inherit this success.

One famous physicist noticed, that all experiments could be quickly explained by "simple, elegant, wrong" theory. But wrong results in astrophysics may lead to great results and discoveries. The best example is a wrong theory of the origin of heavy elements, developed by G. Gamow. Developing this wrong theory he suggested the "big bang" model of the hot universe, and predicted the CMB radiation which was discovered 20 years after and lead to creation of the modern cosmology.

Very important wrong result was obtained by J. Weber in 1968, who claimed the discovery of GW from GC. After many works, disproving his result, the intensive development of the instruments started in different countries, which led to construction of LIGO/VIRGO, on which the real GW discovery should be done (or is done already).

Another example of the wrong result which induced a new branch of investigations is the claim of Saint Petersburg (that time Leningrad) physicists about discovery of the rest mass of the electronic neutrino about $30 \mathrm{eV}$. This result occurs to be wrong, but after its application to the dark 
matter origin, large number of paper appeared with different suggestions about the nature of DM, which was a beginning of Astro-Particle Physics. In the talk of M. Merafina we heard one of the variants of DM nature.

The challenging talk of W. Kundt presented alternative views about many astrophysical results, like collapse, BH, GRB, jet origin etc.. One critical statements in his talk seems to me quite important. The cosmological origin of GRB is based on measurements of the redshifts of optical counterparts. This is done usually on large telescopes, at the time when the optics is weak. In many cases, if not all, the redshifts have been measured for the emission of host galaxies, in which presumably happens the GRB. Due to very dense surface density of galaxies at large redshifts, Kundt suggested that this identification could be wrong, and redshfts are not related to GRB, but only to host. This suggestion could be too strong, but for clear interpretation the spectra should be analysed for the prompt optical emission, coming simultaneously with GRB itself. Outstanding example of the optical prompt emission is obtained by observations of the GRB 080319B, by Russian-Italian experiment TORTORA, using wide field optical instrument (open eye burst).

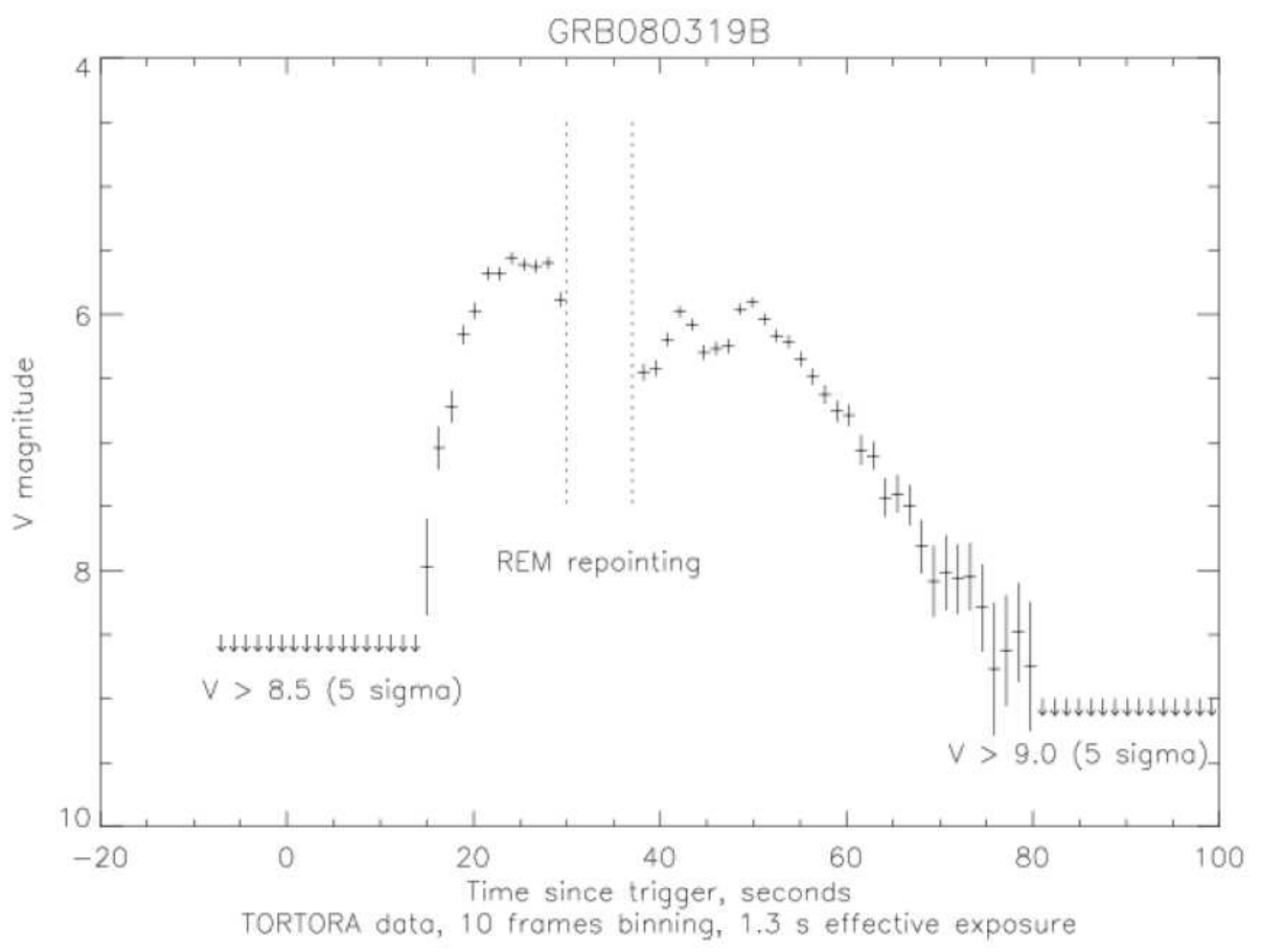

Figure 1: from http://vo.astronet.ru/ karpov/grb080319blc10.gif

The nature of this optical counterpart is still not clear (thermal or non-thermal ), no spectra have been obtained, and no models constructed. Obtaining of spectra and polarization of the prompt optical emission seems to be the most important problem, giving the answer to GRB central machine origin. 
I would like to mention the talk about binary X-ray sources with BH, namely, Cyg X-1, showing different (hard and soft) states. To explain the hard state emission it was suggested to consider accretion disc with hot geometrically tick central region. The author spoke only about the simple Shakura-Sunyaev model, but did not mentioned the model of advective accretion disk (Paczynski, Bisnovatyi-Kogan 1981, Acta Astronomica 31, 283) which gave a possibility to construct a numerical model of advective accretion disk with a smooth transition from the outer optically thick to the inner optically thin, very hot disk (Artemova et al., 2006, ApJ 637, 968), shown in the next two figures from this paper.

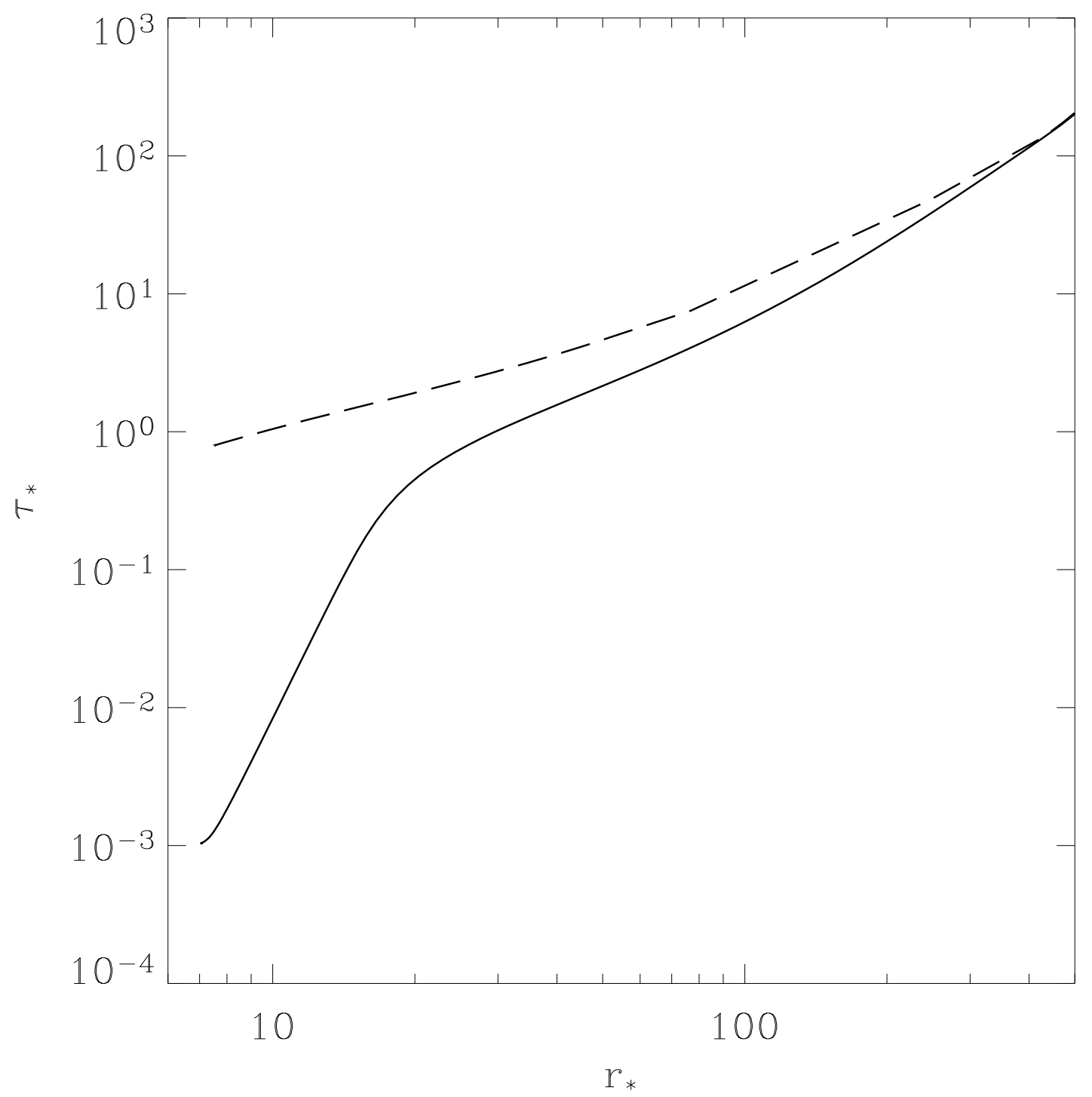

Figure 2: Radial dependence of the effective optical depth $\tau_{*}$

Note that smooth models with optically thin/thick transition do not exist in the local Shakura model, but are characteristic only to advective disks. For parameters of disks with optically thin/thick transition continuous local solutions do not exist, and the solution has unphysical gap 


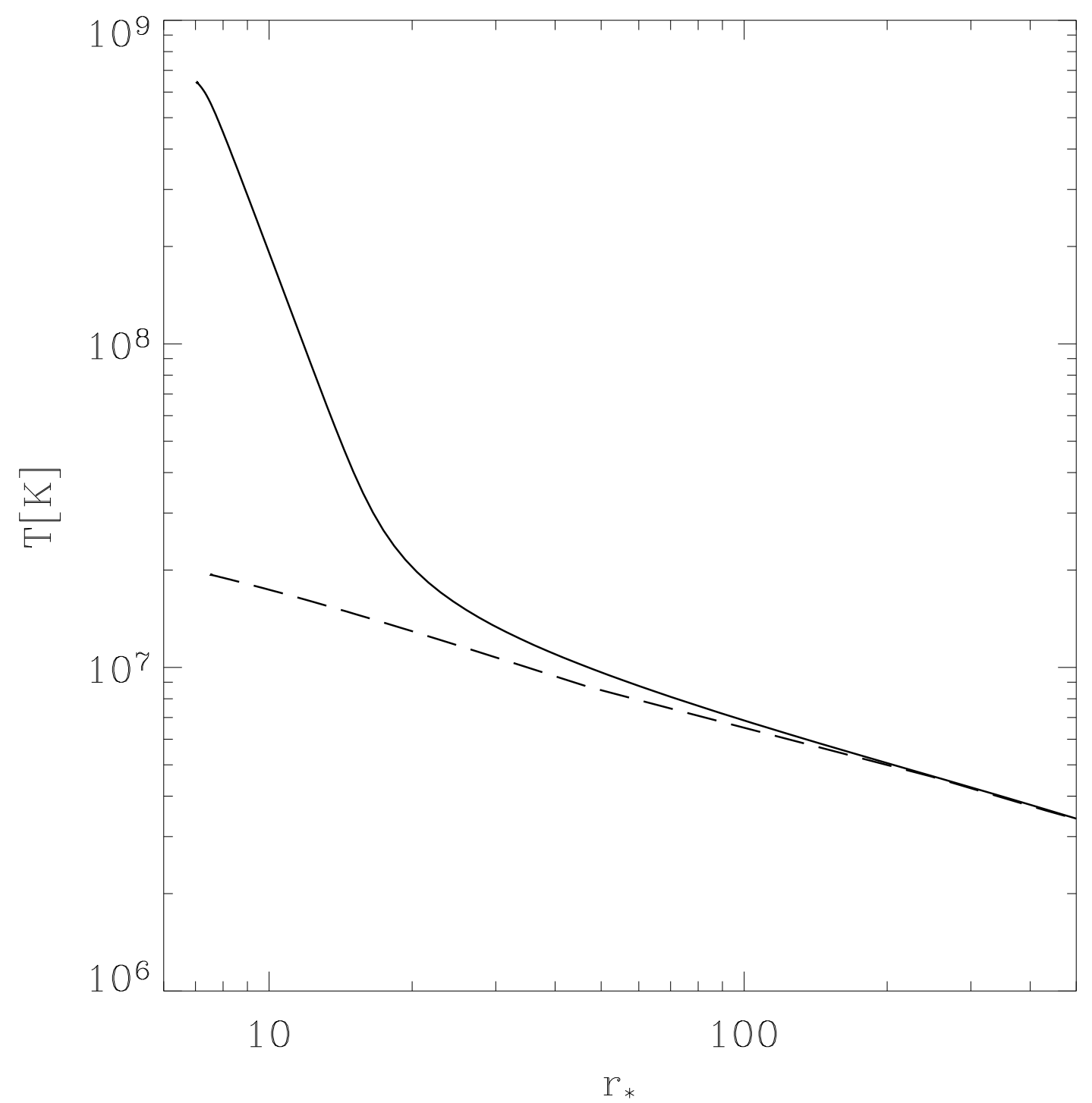

Figure 3: The midplane temperature $T$ for the models with $M_{B H}=10 M_{\odot}, \alpha=0.5$, and $\dot{m}=48$. The dashed lines correspond to the advective optically thick solution and the solid lines correspond to the advective solution with the optical depth transition

when going along the radius..

I express my deep gratitude to the organizers, and first of all, to Franco Giovannelli. 